\title{
BMJ Open Assessment of CardiOvascular Remodelling following Endovascular aortic repair through imaging and computation: the CORE prospective observational cohort study protocol
}

Foeke J H Nauta, ${ }^{1,2}$ Arnoud V Kamman, ${ }^{1,2}$ El-Sayed H Ibrahim, ${ }^{3}$ Prachi P Agarwal, ${ }^{3}$ Bo Yang, ${ }^{4}$ Karen Kim, ${ }^{4}$ David M Williams, ${ }^{3}$ Joost A van Herwaarden, ${ }^{5}$ Frans L Moll, ${ }^{5}$ Kim A Eagle, ${ }^{1}$ Santi Trimarchi, ${ }^{2}$ Himanshu J Patel, ${ }^{4}$ C Alberto Figueroa ${ }^{6}$

To cite: Nauta FJH, Kamman AV, Ibrahim E-SH, et al. Assessment of CardiOvascular Remodelling following Endovascular aortic repair through imaging and computation: the CORE prospective observational cohort study protocol. BMJ Open 2016:6:e12270. doi:10.1136/bmjopen-2016012270

- Prepublication history for this paper is available online. To view these files please visit the journal online (http://dx.doi.org/10.1136/ bmjopen-2016-012270).

Received 12 April 2016 Revised 17 June 2016 Accepted 30 August 2016

CrossMark

For numbered affiliations see end of article.

Correspondence to Dr Foeke J H Nauta; fnauta@ med.umich.edu

\section{ABSTRACT}

Introduction: Thoracic aortic stent grafts are orders of magnitude stiffer than the native aorta. These devices have been associated with acute hypertension, elevated pulse pressure, cardiac remodelling and reduced coronary perfusion. However, a systematic assessment of such cardiovascular effects of thoracic endovascular aortic repair (TEVAR) is missing. The CardiOvascular Remodelling following Endovascular aortic repair (CORE) study aims to (1) quantify cardiovascular remodelling following TEVAR and compare echocardiography against MRI, the reference method; (2) validate computational modelling of cardiovascular haemodynamics following TEVAR using clinical measurements, and virtually assess the impact of more compliant stent grafts on cardiovascular haemodynamics; and (3) investigate diagnostic accuracy of ECG and serum biomarkers for cardiac remodelling compared to MRI.

Methods and analysis: This is a prospective, nonrandomised, observational cohort study. We will use MRI, CT, echocardiography, intraluminal pressures, ECG, computational modelling and serum biomarkers to assess cardiovascular remodelling in two groups of patients with degenerative thoracic aneurysms or penetrating aortic ulcers: (1) patients managed with TEVAR and (2) control patients managed with medical therapy alone. Power analysis revealed a minimum total sample size of 20 patients $(\alpha=0.05$, power $=0.97)$ to observe significant left ventricular mass increase following TEVAR after 1 year. Consequently, we will include 12 patients in both groups. Advanced MRI sequences will be used to assess myocardial and aortic strain and distensibility, myocardial perfusion and aortic flow. ECG, echocardiography and serum biomarkers will be collected and compared against the imaging data. Computational models will be constructed from each patient imaging data, analysed and validated. All measurements will be collected at baseline (prior to TEVAR) and 1-year follow-up. The expected study period is 3 years.

\section{Strengths and limitations of this study}

There are currently no systematic studies on the effects of endovascular aortic repair on cardiovascular remodelling.

- The results will serve as a guide for clinicians, stent-graft designers and researchers to continuously press the need for improvement of stent grafts.

- As a single-centre, nonrandomised, observational cohort study, generalisability of results may be limited; this will require replication at other centres and patients populations.

Ethics and dissemination: This study has been approved by the University of Michigan IRB. The results will be disseminated through scientific journals and conference presentations.

Trial registration number: NCT02735720.

\section{INTRODUCTION}

\section{Background}

Thoracic endovascular aortic repair (TEVAR) entails the implantation of a stiff stent graft in a diseased or ruptured thoracic aorta. The use of TEVAR is increasing rapidly and is being employed in younger patients. ${ }^{12}$ For instance, the rate of TEVAR for descending thoracic aortic aneurysm (TAA) in Great Britain has almost tripled between 2006 and 2010, from 0.45 to 1.27 cases per 100000 , and more than doubled for type $\mathrm{B}$ aortic dissection from 0.22 to 0.50 cases per $100000 .^{2}$

Current stent grafts have biomechanical properties that are several orders of magnitude stiffer than the native aorta. ${ }^{3}$ The elastic 
modulus of the thoracic aorta in a middle-aged participant is $0.44 \mathrm{MPa}$. Conversely, a typical stent graft has an elastic modulus of $55.2 \mathrm{MPa}^{3}{ }^{4}$ The impact of such a stiff device on cardiovascular function has not yet been elucidated, despite the critical role of aortic elasticity in damping the highly pulsatile flow entering the aorta from the left ventricle (LV). The elastic aorta stores flow during systole and releases it in diastole. This phenomenon, known as the 'Windkessel effect', results in a more continuous flow pattern to the distal vascular beds throughout the cardiac cycle. Alterations in aortic stiffness have been linked to disease. ${ }^{5-7}$ The aorta stiffens naturally with age and also with smoking, high cholesterol levels, genetic predisposition and stent grafting. ${ }^{8-10}$ Pre-clinical and clinical studies have reported acute aortic stiffening following TEVAR resulting in hypertension, elevated pulse pressure, reduced coronary perfusion and adverse cardiovascular remodelling leading ultimately to heart failure ${ }^{9-11}$ However, these studies have important limitations such as animal models not directly translatable to humans, retrospective design, use of echocardiography alone and mixed (eg, abdominal and thoracic aneurysms) patient populations.

We next provide an overview of several modalities used to quantify cardiac remodelling. ECG is the most commonly used non-invasive test to assess cardiac structure and function. However, this test has low sensitivity in detecting LV hypertrophy. ${ }^{12}{ }^{13}$ Nevertheless, it should be noted that the combined use of ECG and serum cardiac biomarkers has shown beneficial results, which may offer an easily available, minimally invasive diagnostic and prognostic test for cardiac remodelling. ${ }^{14} 15$

\section{Echocardiography}

This is widely used to study cardiac remodelling. ${ }^{16}{ }^{17}$ Takeda $e t a t^{\ominus}$ found an increase in LV mass and left atrial volume following endovascular aortic repair through echocardiography. Recently, our research group retrospectively investigated cardiac remodelling following TEVAR using echocardiography. We enrolled all TAA patients managed with TEVAR at our centre between 2012 and 2015. Patients with severe heart or heart valve disease were excluded. Preoperative and postoperative (1 year follow-up) echocardiography and CT data were available for 6 patients. We observed an average $\mathrm{LV}$ mass increase of $39 \%(n=6, p=0.047)$ at follow-up. This motivates further research with larger sample size and higher quality imaging since echocardiography is associated with low reproducibility and high operatordependency, even when using three-dimensional echocardiography. ${ }^{17} 18$

\section{Cardiac MRI}

This is the gold standard to assess cardiac remodelling due to its high resolution and tissue contrast, high accuracy, lack of ionising radiation, and plethora of functional, structural and anatomical parameters that can be used to evaluate the heart condition. ${ }^{18}{ }^{19}$ It has been shown that MRI has significantly higher reproducibility and lower operator-dependency than echocardiography for diagnosing cardiac remodelling. ${ }^{18}$ Modern sequences such as MRI Tagging, strain-encoding (SENC) and Steady State Free Precession provide detailed quantification of cardiac and aortic strain and distensibility, and first-pass perfusion contrast-enhanced MRI can assess coronary perfusion. ${ }^{20-23}$ Moreover, phase contrast (PC) MRI offers a non-invasive technique to measure pulse wave velocity (PWV) along a particular segment of interest. ${ }^{24}$ Using cardiac MRI as research imaging modality may be challenging as it requires local expertise, longer examination time and higher costs than echocardiography. In 2011, our group studied the relationship between aortic stiffness and diastolic dysfunction in patients with heart failure with normal ejection fraction (HFNEF) compared to controls using MRI. Aortic stiffness was assessed using PWV via PC MRI and the transit time method. Myocardial regional function was evaluated by tissue strain via MRI tagging, and LV diastolic function was assessed by the early-to-late atrial filling ratio (E/A) (figure 1). ${ }^{20}$ The results showed that aortic PWV was higher in HFNEF than in volunteers due to reduced vessel compliance. The $\mathrm{E} / \mathrm{A}$ ratio and myocardial strain measurements showed inverse correlations.

\section{Computational Fluid Dynamics}

Recent developments in computational fluid dynamics (CFD) are among the most promising means to understand the effects of regional variations in material properties and arterial geometry on local and systemic haemodynamics. ${ }^{25}$ A preliminary CFD study assessing the impact of systemic (ie, ageing) and localised stiffening (ie, ascending aortic stent-graft repair) on cardiac function has been recently performed by our group. The study demonstrated that increases in LV contractility $(20 \%$ for the ageing, $8.5 \%$ for the stent-graft repair) and work (11\% for the ageing, $5 \%$ for the stent-graft repair) are required to maintain baseline cardiac output (figure 2). These results highlight that alterations in stiffness in a relatively short segment of the aorta have comparable impacts on those resulting from generalised age-induced stiffening.

To date, the impact of stent grafts on cardiovascular remodelling has not been assessed systematically. The lack of qualitative data presses the need for prospective, well-designed, controlled research to study such impact, which is the purpose of this protocol.

\section{Objectives and hypotheses}

Our objectives are

1. To quantify cardiovascular remodelling following TEVAR through MRI and intraluminal pressure measurements. Echocardiography will be compared against MRI to assess its diagnostic accuracy. 

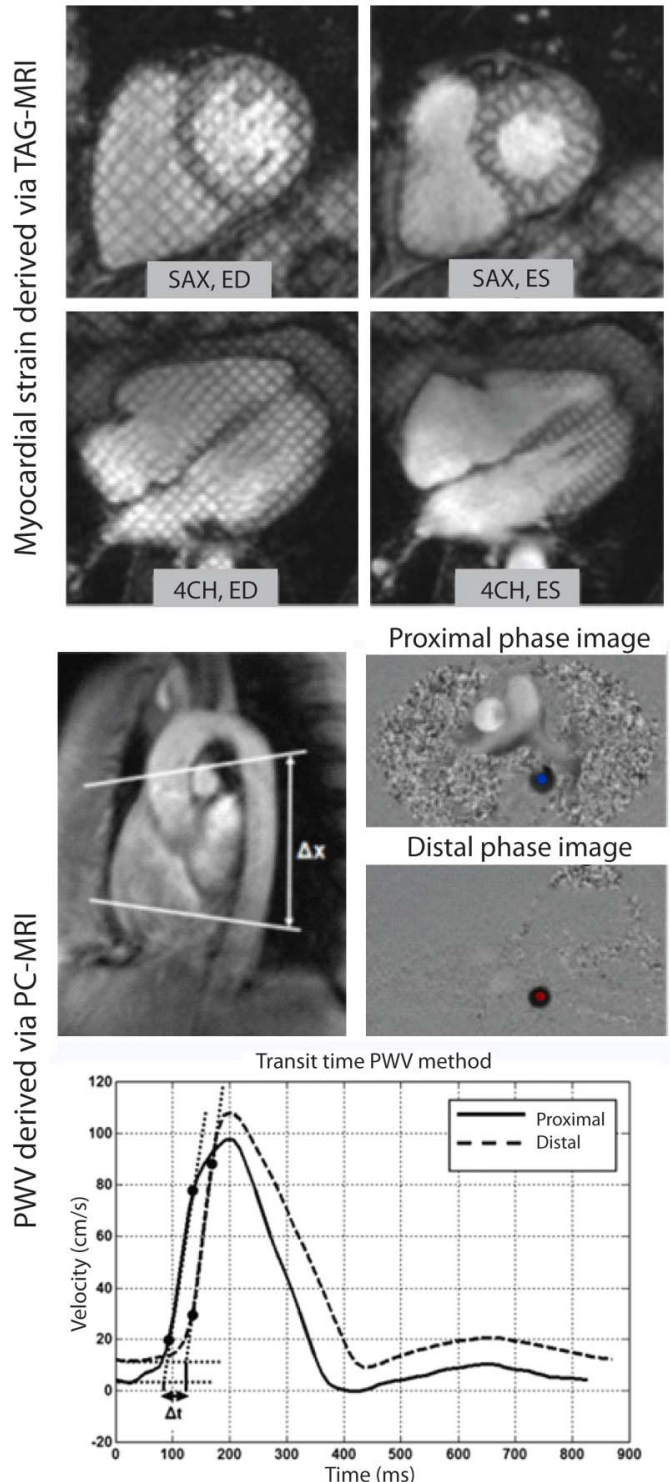

Figure 1 Top: MRI Tagging sequence to quantify myocardial strain. SAX and $4 \mathrm{CH}$ tagged images at ED and ES. Bottom: Transit time $(\Delta t)$ between proximal and distal flow waveforms measured with PC MRI sequences is used to evaluate PWV in the aorta. ${ }^{20} 4 \mathrm{CH}$, four-chamber; $\mathrm{ED}$, end diastole; $\mathrm{ES}$, end systole; SAX, short axis.

2. To investigate the diagnostic accuracy of ECG and serum biomarkers for evaluating cardiac remodelling compared to MRI, the reference method.

3. To validate computational modelling of thoracic aortic haemodynamics following TEVAR using the acquired clinical measurements. Once validated, computational analyses will be performed to virtually assess the impact of more compliant stent grafts on cardiac and aortic haemodynamics.

The ultimate goal of this study is to protect patients from potential unknown adverse effects of TEVAR, thus improving TEVAR-related outcomes. We hypothesise that TEVAR stiffens the thoracic aorta resulting in adverse cardiovascular remodelling, while more compliant stent grafts reduce this impact.

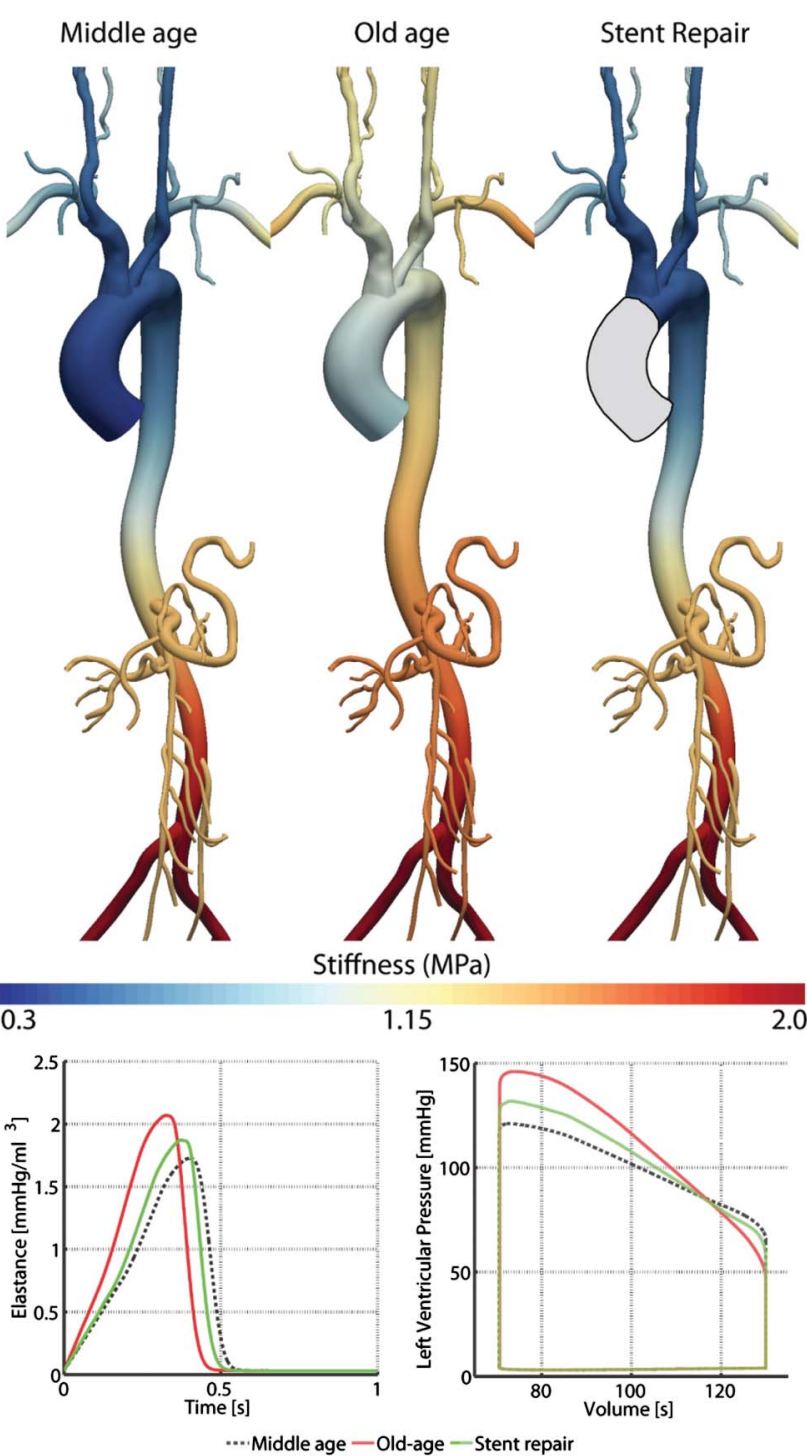

Figure 2 Top: Arterial stiffness for a baseline 'middle-age' participant, an 'old-age' participant showing generalised arterial stiffening and a 'stent repair' case with identical stiffness to the baseline except for the ascending aortic segment, in which a stent graft (presented in grey), whose stiffness is $125 \times$ higher than the native aortic tissue, was inserted. Bottom: Changes in LV function required to maintain cardiac output following generalised (old age) or stent-induced stiffening. Left: changes in cardiac contractility, as given by a LV elastance function. Right: changes in LV work, estimated by computed pressure-volume loops (unpublished data Lau et al 2016). LV, left ventricle.

\section{METHODS AND ANALYSIS}

Study design and settings

This is a single-centre, prospective, nonrandomised, observational cohort study conducted at the Frankel Cardiovascular Center at the University of Michigan Health System, a large regional hospital with expertise in managing aortic disease. All consecutive patients with descending degenerative TAA or penetrating aortic ulcers (PAU) will be evaluated by the cardiac surgeon in 
charge and, if deemed eligible, invited to take part in the study.

\section{Participants}

The patient population will consist of adult patients (aged 18 years or older) with descending degenerative TAA or with PAU managed with TEVAR (TEVAR group, $\mathrm{n}=12$ ) or with optimal medical treatment alone (control group, $n=12$ ). Choice of management will be left to the discretion of the care-taking physician, as well as the size and manufacturer of the stent graft used. Patients will be excluded if they present with any of the following conditions: ejection fraction $<35 \%$; LV wall motion abnormality; poor renal function (estimated glomerular filtration rate (eGFR) $<60 \mathrm{~mL} / \mathrm{min} / 173 \mathrm{~m}^{2}$ ); pregnancy; connective tissue disorder; significant valve, lung or congenital heart disease; history of cardiac or aortic surgery; expected cardiac or aortic surgery within the study period; and standard MRI contraindications (pacemakers, non-compatible metal implants and claustrophobia).

\section{Procedures and standard care}

The study flow chart is illustrated in figure 3 .

\section{TEVAR group}

Standard work-up examinations for TEVAR will be collected, consisting of CT, echocardiography, brachial blood pressure, heart rate, ECG and blood tests. Once consent is confirmed, and prior to the TEVAR procedure (window of 0-30 days), a non-invasive MRI scan and blood samples will be acquired in addition to the standard of care measurements. Intraluminal pressure measurements will be collected during the TEVAR procedure. One year (window of 275-455 days) following TEVAR, the participant will undergo a second MRI study in addition to the standard clinical imaging follow-up CT scan. Measurements of brachial blood pressure, heart rate, ECG and blood testing will also be repeated at follow-up.

\section{Control group}

Patients with stable degenerative TAA or PAU not requiring aortic repair are monitored in the outpatient clinic as standard of care. We will collect standard-of-care measurements on brachial blood pressures and heart rates in these patients. In addition, these participants will undergo an ECG, blood testing and one MRI scan at baseline. One year (window of 275-455 days) after baseline, this group will undergo a second MRI study, with subsequent brachial blood pressure and heart rate measurements, ECG and blood testing.

\section{Blood testing}

We will acquire the following cardiac biomarkers: B-type natriuretic peptide (BNP), N-terminal of the prohormone brain natriuretic peptide (NT-pro-BNP), and Troponin T. In addition, standard blood tests will be collected, which include complete blood count with platelets (ie, white cell counts, haemoglobin, haematocrit and red blood cells) and a basic metabolic panel (including sodium, potassium, glucose and calcium).

\section{Echocardiography}

Preoperative echocardiography is standard of care in all patients requiring TEVAR. The obtained echocardiographic measurements ( $\mathrm{LV}$ mass index, left atrial volume index and E/A filling ratios) will be collected and compared to the preoperative MRI data.

\section{Intraluminal pressure}

During TEVAR, an angiography will be performed as standard of care. Intraluminal pressure measurements will be conducted in the TEVAR group, using the catheters and guidewires that will be already in place for the deployment of the stent graft, as is common practice at the University of Michigan Health System.

\section{Magnetic resonance imaging}

Table 1 summarises the MRI sequences used in this study, which are approved either by FDA or by IRB. The protocol will be built and imaging parameters optimised by E.I.

PC MRI measurements will be acquired at different levels of the thoracic aorta using through-plane velocity encoding. Images will also be captured in an oblique sagittal view (in-plane velocity encoding) to estimate the aortic vessel wall stiffness via PWV measurements. PC MRI images will be acquired across the mitral valve to evaluate the LV diastolic function through the measurement of $\mathrm{E} / \mathrm{A}$ filling ratios. Measurements from the cine images will include LV volume, LV mass and left atrial volume. MRI Tagging or SENC will be used to measure different components of myocardial strain in standard short-axis and four-chamber views.
Figure 3 Patient population and clinical measurements of our study. BP, blood pressure; HR, heart rate.

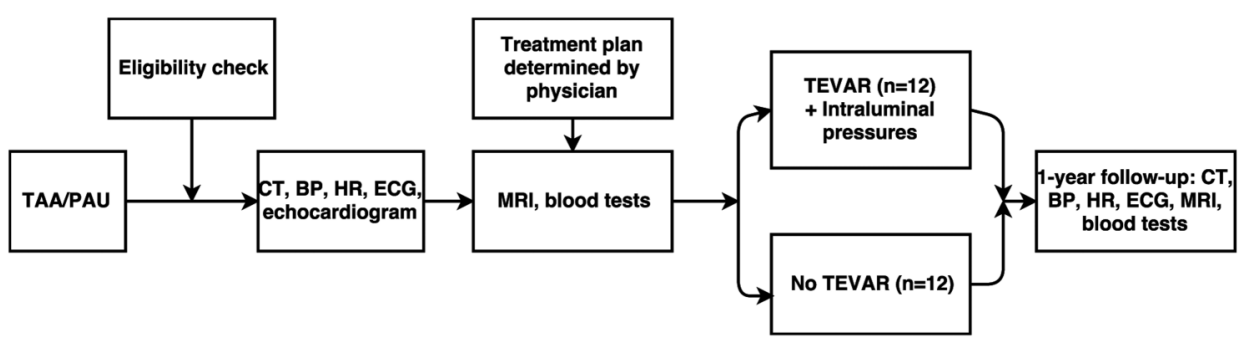


Table 1 Overview of performed MRI sequences

\begin{tabular}{|c|c|c|c|c|}
\hline Sequence & Description & Analysis & Variables measured & $\begin{array}{l}\text { Estimate } \\
\text { time }\end{array}$ \\
\hline $\begin{array}{l}\text { Cine Steady State Free } \\
\text { Precession or } \\
\text { gradient-refocused echo }\end{array}$ & $\begin{array}{l}\text { A stack of cine short-axis images covering } \\
\text { the whole heart, as well as 4-chamber and } \\
\text { 2-chamber images, will be acquired. }\end{array}$ & $\begin{array}{l}\text { A radiologist }(\mathrm{PA}) \text { will analyse the } \\
\text { images using Medis QMass software. } \\
\text { The endo- and epicardial ventricular } \\
\text { boundaries will be determined to } \\
\text { measure ventricular volumes, function } \\
\text { and mass, as well as left atrial volume. }\end{array}$ & $\begin{array}{l}\text { Ventricular volumes and myocardial } \\
\text { mass to evaluate ventricular dilation and } \\
\text { hypertrophy (all measurements will be } \\
\text { normalised by the patient's body surface } \\
\text { area). Left atrial volume. }\end{array}$ & $15 \mathrm{~min}$ \\
\hline Trans-mitral VENC & $\begin{array}{l}\text { Trans-mitral PC velocity-encoded flow } \\
\text { images will be acquired. VENC parameter } \\
\text { setting will be adjusted to avoid velocity } \\
\text { aliasing. }\end{array}$ & $\begin{array}{l}\text { PA will analyse the images using Medis } \\
\text { QFlow software. The mitral valve } \\
\text { boundary will be determined through all } \\
\text { phases, from which E/A ventricular } \\
\text { filling ratio, will be determined. }\end{array}$ & $\begin{array}{l}\text { Ventricular early-to-atrial filling ratio, as a } \\
\text { measure of ventricular diastolic function. }\end{array}$ & $2-3 \min$ \\
\hline VENC across the aorta & $\begin{array}{l}\text { VENC PC flow images will be acquired at } 4 \\
\text { different locations (ascending aorta, aortic } \\
\text { arch branches, proximal descending aorta } \\
\text { and at the level of the diaphragm). VENC } \\
\text { setting will be adjusted to avoid velocity } \\
\text { aliasing. }\end{array}$ & $\begin{array}{l}\text { PA will analyse the images using Medis } \\
\text { QFlow software. The cross section of } \\
\text { the aorta will be determined through all } \\
\text { phases, from which velocity and flow, } \\
\text { will be determined. Further, El will } \\
\text { analyse the images using in-house } \\
\text { software developed with Matlab to } \\
\text { measure PWV. }\end{array}$ & $\begin{array}{l}\text { Blood flow and velocity patterns at } \\
\text { different cross sections of the aorta, as } \\
\text { well as PWV as a measure of aortic } \\
\text { stiffness. }\end{array}$ & $\begin{array}{l}25 \mathrm{~min} \\
\text { (total) }\end{array}$ \\
\hline $\begin{array}{l}\text { SENC/MRI Tagging (IRB } \\
\text { approved) }\end{array}$ & $\begin{array}{l}\text { SENC or conventional tagged images will } \\
\text { be acquired at the basal, mid-ventricular } \\
\text { and apical short-axis slices, as well as in a } \\
\text { 4-chamber slice. }\end{array}$ & $\begin{array}{l}\text { An MRI physicist }(\mathrm{EI}) \text { will analyse the } \\
\text { images using Diagnosoft software to } \\
\text { measure myocardial strain. }\end{array}$ & Regional myocardial strain. & $10 \min$ \\
\hline Perfusion & $\begin{array}{l}\text { Myocardial perfusion images will be } \\
\text { acquired after administration of } \\
\text { gadolinium-based contrast agent under } \\
\text { pharmacological stress. }\end{array}$ & $\begin{array}{l}\text { PA will analyse the images visually and } \\
\text { using Medis QFlow software to } \\
\text { determine any perfusion defects as } \\
\text { well as the myocardium contrast uptake } \\
\text { and washout curves. }\end{array}$ & $\begin{array}{l}\text { Myocardial perfusion defects, and } \\
\text { contrast uptake and washout patterns. }\end{array}$ & $10 \mathrm{~min}$ \\
\hline
\end{tabular}


SENC sequences will measure cardiac and aortic strains. Cine images will be used to measure strain of the thoracic aorta through measurements in the ascending aorta just distal to the coronary branches and proximal to the coeliac trunk. The sequence is obtained in accordance with FDA safety guidelines. The total anticipated scan time is $\sim 1$ hour. We will use a $3 \mathrm{~T}$ scanner to gain from the double signal-to-noise ratio provided by this higher field strength compared to $1.5 \mathrm{~T}$ scanners. Interobserver and intraobserver variability analyses (with P.A. and E.I. as observers) will be computed to validate reproducibility.

\section{Computational fluid dynamics}

CFD has been used profusely in the past decade for the investigation of haemodynamics in complex anatomical models built from image data. The so-called imagebased modelling' paradigm uses anatomical data (obtained from CT or MRI images) to create a 3D computer model representing the blood vessels of interest where the simulation of haemodynamics will be performed. Additional physiological measurements of flow, pressure or wall motion are used to inform the 'boundary conditions' of the simulation. Computational analyses, usually requiring high-performance computing hardware, are performed, and detailed descriptions of haemodynamic quantities such as velocity, pressure and wall shear stress are obtained for the entire volume of interest. CFD techniques have been recently approved by the FDA for non-invasive assessment of coronary stenosis. $^{26}$

Using the imaging and clinical data, simulations of blood flow and pressure will be performed in the High-Performance Computer cluster 'Flux' at the University of Michigan. The parameters of the simulations will be iteratively adjusted until clinical data on flow, pressure and wall motion are matched within $\pm 5 \%$. Highly detailed descriptions of velocity, flow, pressure, wall shear stress and other haemodynamically significant quantities will be obtained. Once the models are validated against clinical data, additional computational analyses will be performed to virtually assess the impact of more compliant stent grafts on cardiac and aortic haemodynamics.

\section{Outcome measures}

\section{Primary outcome measures}

The primary outcomes are LV mass, E/A ratios, aortic flow (in the ascending aorta, all arch branches, proximal descending aorta and distal descending aorta), myocardial and aortic strain, central aortic PWV and myocardial perfusion.

\section{Secondary outcome measures}

The secondary outcome measures are as follows: diagnostic accuracy for cardiac remodelling of ECG alone, as well as in combination with BNP, NT-pro-BNP and Troponin $\mathrm{T}$ compared to MRI; validation of computational modelling of thoracic aortic haemodynamics following TEVAR against intraluminal pressures and MRI; and performance of more compliant stent-graft designs on aortic haemodynamics.

\section{Statistical methods}

Sample size calculation

A sample size calculation was performed for the primary outcome: significant LV mass increase within 1 year following TEVAR. Our preliminary echocardiographic data of TAA patients treated with TEVAR at the University of Michigan Health System (Nauta et al, unpublished data, 2016) revealed an average LV mass increase of $39 \%$ $(p=0.047)$ at 1-year follow-up. Power analysis for a Mann-Whitney U test, based on these preliminary data, revealed that for a significant threshold of $5 \%(\mathrm{p}<0.05$, two-sided test), a total sample size of 20 patients ( $\alpha=0.05$, power $=97 \%$ ) would be needed to observe a significant effect of TEVAR on LV mass increase. To ensure a margin of error, we aim to include 12 TEVAR patients and 12 control patients.

\section{Statistical analysis}

Statistical analysis will include descriptive and comparative studies of clinical measurements and data acquired from computational modelling. We will assign patients to either the TEVAR or control group. Subanalysis will include grouping by stent-graft type and length, gender and age. The patients identifying information and protected health data will be dealt with according to Health Insurance Portability and Accountability Act (HIPAA) guidelines. Categorical variables will be compared using the $\chi^{2}$ test or Fisher's exact test, and continuous variables will be analysed using Student's t-test or the Mann-Whitney U test, as appropriate. Log-rank tests will be performed to compare between-group differences. Changes in haemodynamics will be evaluated by Wilcoxon's signed rank testing. A $p$ value of $<0.05$ will be considered significant.

\section{Ethics and dissemination \\ Assessment of risks}

This study adds little risk to the standard of care for these patients. The main added risks include blood testing, use of a gadolinium-based contrast agent and intraluminal pressure measures. Gadolinium is associated with a minor risk $(\sim 2 \%)$ of headache, nausea, hives, temporary low blood pressure and rarer side effects including renal failure and allergic reactions. Intraluminal pressure measurement carries nearly no additional risks since the guidewires will already be in place for the TEVAR procedure.

\section{Informed consent}

Consent will be obtained during standard clinical visits required for surgery or follow-up for medical management. The patient and his/her family members, if present, will be given information about the study and 
any alternative treatments that are available. The informed consent process will be documented and attached to the signed informed consent document. A copy will be given to the patient and uploaded in the patients' electronic dossier. The original, signed hardcopy will be kept in the subject binder.

\section{Confidentiality/security}

The study team can access data linked to a participant's identity. The patient privacy will be protected by a secured database that will be maintained and saved in a separate location from the research records. Only those directly involved with this study will have access to the records. The research records, data and specimens will be protected against inappropriate use or disclosure, or malicious or accidental loss or destruction by keeping them in a locked office with restricted access. Blood samples will be processed, analysed and then destroyed. Furthermore, there will be restrictions on copying studyrelated materials. All digital data will be kept on secure laptops, with individual ID and password protection and encryption of digital data.

No data will be generated that, if revealed, might place the participants at risk of personal safety, criminal or civil liability, or damage to their financial standing, employability or reputation, and no data will be provided to a repository as part of a data-sharing agreement. At the end of the study, data will be retained for record-keeping purposes for 7 years.

Patients are identified by direct identifiers stored on data records (eg, name, initials, phone number or medical record number) and indirect identifiers including a link to direct identifiers. This is necessary because the data must be linked to the patient for verification purposes. Link lists will be maintained and saved in a separate location from the research records. Only those directly involved with the study will have access to the records. No individually identifiable sensitive information will be disclosed.

\section{DISCUSSION}

The CardiOvascular Remodelling following Endovascular aortic repair (CORE) study will systematically assess the effects of TEVAR on cardiovascular function through a unique data set consisting of serum biomarkers, imaging, intraluminal pressure measurements and computational modelling. This research is timely and clinically important since TEVAR is being performed increasingly and long-term effects remain undetermined. Detailed data on the cardiovascular effects of stent grafting, such as provided by the CORE study, are needed since its practice may extend towards the aortic arch and ascending aorta in the near future, where the stiffening impact could be more profound. Moreover, the stiffening effects of current stent grafts may be of particular importance in young patients as their hearts and aortas are more compliant than those of older patients. This calls for further evaluation since TEVAR is performed increasingly in young patients. Future aortic patients might benefit from data provided by the CORE study through improved aortic stent-graft designs and long-term outcomes.

As a single-centre, nonrandomised, observational cohort study, generalisability of results may be limited, which will require replication at other centres and patient populations. Moreover, potential loss of follow-up may lead to a prolonged study period as such patients will be excluded from the study. Control participants managed with aortic repair during the follow-up period may also lead to exclusion (in case of open surgery) or to a switch to the TEVAR group, which might increase the study period. Moreover, stent grafts of different manufacturers will be used. Therefore, care should be taken when translating our findings to devices other than those used in this study. Finally, pressures in our CFD models of control participants will rely exclusively on non-invasive brachial pressure data, while models of TEVAR patients will also rely on intraluminal pressure data measured by a catheter.

\section{Author affiliations}

${ }^{1}$ Cardiovascular Center, University of Michigan Health System, Ann Arbor, Michigan, USA

${ }^{2}$ Department of Vascular Surgery, Thoracic Aortic Research Center, Policlinico San Donato IRCCS, University of Milan, Milan, Italy

${ }^{3}$ Department of Radiology, University of Michigan, Ann Arbor, Michigan, USA ${ }^{4}$ Department of Cardiac Surgery, University of Michigan, Ann Arbor, Michigan, USA

${ }^{5}$ Department of Vascular Surgery, University of Utrecht, Utrecht, MiddenNederland, The Netherlands

${ }^{6}$ Departments of Biomedical Engineering and Surgery, University of Michigan, Ann Arbor, Michigan, USA

Contributors FJHN, JAvH, FLM, ST and CAF conceived of the study. FJHN, E-SHI, PPA, JAvH, FLM, KAE, HJP and CAF planned the study. FJHN, AVK, E-SHI, PPA, BY, KK, DMW, HJP and CAF will be responsible for data collection, quality analysis and storage. The initial draft of the present manuscript was created by FJHN and AVK and circulated among the authors for critical revision. All authors approved the final version of the manuscript.

Funding This work was supported by the University of Michigan Frankel Cardiovascular Center, Ann and Bob Aikens, the Edward B. Diethrich M.D. professorship, Joe D. Morris Professorship, David Hamilton Fund and Phil Jenkins Breakthrough Fund, and the European Research Council under the European Union's Seventh Framework Programme (FP/2007-2013) with grant number 307532.

\section{Competing interests None declared.}

Ethics approval University of Michigan Institutional Review Board.

Provenance and peer review Not commissioned; externally peer reviewed.

Open Access This is an Open Access article distributed in accordance with the Creative Commons Attribution Non Commercial (CC BY-NC 4.0) license, which permits others to distribute, remix, adapt, build upon this work noncommercially, and license their derivative works on different terms, provided the original work is properly cited and the use is non-commercial. See: http:// creativecommons.org/licenses/by-nc/4.0/

\section{REFERENCES}

1. Perera $\mathrm{AH}$, Rudarakanchana N, Hamady M, et al. New-generation stent grafts for endovascular management of thoracic 
pseudoaneurysms after aortic coarctation repair. J Vasc Surg 2014;60:330-6.

2. von Allmen RS, Anjum A, Powell JT. Incidence of descending aortic pathology and evaluation of the impact of thoracic endovascular aortic repair: a population-based study in England and Wales from 1999 to 2010. Eur J Vasc Endovasc Surg 2013;45:154-9.

3. Kleinstreuer C, Li Z, Basciano CA, et al. Computational mechanics of Nitinol stent grafts. J Biomech 2008;41:2370-8.

4. Roccabianca S, Figueroa CA, Tellides G, et al. Quantification of regional differences in aortic stiffness in the aging human. $J$ Mech Behav Biomed Mater 2014;29:618-34.

5. Kingwell BA, Waddell TK, Medley TL, et al. Large artery stiffness predicts ischemic threshold in patients with coronary artery disease. J Am Coll Cardiol 2002;40:773-9.

6. Redheuil A, Wu CO, Kachenoura N, et al. Proximal aortic distensibility is an independent predictor of all-cause mortality and incident CV events: the MESA study. J Am Coll Cardiol 2014;64:2619-29.

7. Laurent S, Zanchetti A. New challenges in the disease management of hypertension. J Hypertens Suppl 2001;19:S1.

8. Dobson G, Flewitt J, Tyberg JV, et al. Endografting of the descending thoracic aorta increases ascending aortic input impedance and attenuates pressure transmission in dogs. Eur $J$ Vasc Endovasc Surg 2006;32:129-35.

9. Takeda $\mathrm{Y}$, Sakata $\mathrm{Y}$, Ohtani $\mathrm{T}$, et al. Endovascular aortic repair increases vascular stiffness and alters cardiac structure and function. Circ J 2014;78:322-8.

10. Tzilalis VD, Kamvysis D, Panagou P, et al. Increased pulse wave velocity and arterial hypertension in young patients with thoracic aortic endografts. Ann Vasc Surg 2012;26:462-7.

11. Watanabe $\mathrm{H}$, Ohtsuka $\mathrm{S}$, Kakihana $\mathrm{M}$, et al. Coronary circulation in dogs with an experimental decrease in aortic compliance. J Am Coll Cardiol 1993;21:1497-506.

12. Alfakih K, Walters $\mathrm{K}$, Jones $\mathrm{T}$, et al. New gender-specific partition values for ECG criteria of left ventricular hypertrophy: recalibration against cardiac MRI. Hypertension 2004;44:175-9.

13. Devereux RB, Koren MJ, de Simone G, et al. Methods for detection of left ventricular hypertrophy: application to hypertensive heart disease. Eur Heart J 1993;14(Suppl D):8-15.

14. Courand PY, Gaudebout N, Mouly-Bertin C, et al. Biological electrical and echocardiographic indices versus cardiac magnetic resonance imaging in diagnosing left ventricular hypertrophy. Hypertens Res 2014;37:444-51.

15. Pastormerlo LE, Agazio A, Benelli E, et al. Usefulness of high-sensitive troponin elevation after effort stress to unveil vulnerable myocardium in patients with heart failure. Am J Cardiol 2015;116:567-72.

16. Lang RM, Bierig M, Devereux RB, et al. Recommendations for chamber quantification: a report from the American Society of
Echocardiography's Guidelines and Standards Committee and the Chamber Quantification Writing Group, developed in conjunction with the European Association of Echocardiography, a branch of the European Society of Cardiology. J Am Soc Echocardiogr 2005;18:1440-63.

17. Dorosz JL, Lezotte DC, Weitzenkamp DA, et al. Performance of 3-dimensional echocardiography in measuring left ventricular volumes and ejection fraction: a systematic review and meta-analysis. J Am Coll Cardiol 2012;59:1799-808.

18. Grothues F, Smith GC, Moon JC, et al. Comparison of interstudy reproducibility of cardiovascular magnetic resonance with two-dimensional echocardiography in normal subjects and in patients with heart failure or left ventricular hypertrophy. $A m$ J Cardiol 2002;90:29-34.

19. Bogaerts J. Clinical Cardiac MRI. Springer-Verlag Berlin Heidelberg 2nd edn. 2012.

20. Ibrahim el SH, Miller AB, White RD. The relationship between aortic stiffness and E/A filling ratio and myocardial strain in the context of left ventricular diastolic dysfunction in heart failure with normal ejection fraction: insights from magnetic resonance imaging. Magn Reson Imaging 2011;29:1222-34.

21. Papanastasiou G, Williams MC, Kershaw LE, et al. Measurement of myocardial blood flow by cardiovascular magnetic resonance perfusion: comparison of distributed parameter and Fermi models with single and dual bolus. J Cardiovasc Magn Reson 2015;17:17.

22. Mordini FE, Haddad T, Hsu LY, et al. Diagnostic accuracy of stress perfusion CMR in comparison with quantitative coronary angiography: fully quantitative, semiquantitative, and qualitative assessment. JACC Cardiovasc Imaging 2014;7:14-22.

23. Ko SM, Hwang HK, Kim SM, et al. Multi-modality imaging for the assessment of myocardial perfusion with emphasis on stress perfusion CT and MR imaging. Int J Cardiovasc Imaging 2015;31 (Suppl 1):1-21.

24. Ibrahim el SH, Johnson KR, Miller AB, et al. Measuring aortic pulse wave velocity using high-field cardiovascular magnetic resonance: comparison of techniques. J Cardiovasc Magn Reson 2010;12:26.

25. Coogan JS, Humphrey JD, Figueroa CA. Computational simulations of hemodynamic changes within thoracic, coronary, and cerebral arteries following early wall remodeling in response to distal aortic coarctation. Biomech Model Mechanobiol 2013;12:79-93.

26. FDA. FDA News Release FDA allows marketing of non-invasive device to help evaluate heart blood flow [Internet]. Secondary FDA News Release FDA allows marketing of non-invasive device to help evaluate heart blood flow [Internet]. 2014. http://www.fda.gov/ NewsEvents/Newsroom/PressAnnouncements/ucm424945.htm 